\title{
Memory trace stabilization leads to large-scale changes in the retrieval network: A functional MRI study on associative memory
}

\author{
Atsuko Takashima, ${ }^{1,3}$ Ingrid L.C. Nieuwenhuis, ${ }^{1}$ Mark Rijpkema, ${ }^{1}$ \\ Karl Magnus Petersson, ${ }^{1}$ Ole Jensen, ${ }^{1}$ and Guillén Fernández ${ }^{1,2}$ \\ ${ }^{1}$ FC Donders Centre for Cognitive Neuroimaging, Radboud University Nijmegen, 6500 HB Nijmegen, The Netherlands; \\ ${ }^{2}$ Department of Neurology, Radboud University Nijmegen, 6500 HB Nijmegen, The Netherlands
}

\begin{abstract}
Spaced learning with time to consolidate leads to more stabile memory traces. However, little is known about the neural correlates of trace stabilization, especially in humans. The present fMRI study contrasted retrieval activity of two well-learned sets of face-location associations, one learned in a massed style and tested on the day of learning (i.e., labile condition) and another learned in a spaced scheme over the course of one week (i.e., stabilized condition). Both sets of associations were retrieved equally well, but the retrieval of stabilized association was faster and accompanied by large-scale changes in the network supporting retrieval. Cued recall of stabilized as compared with labile associations was accompanied by increased activity in the precuneus, the ventromedial prefrontal cortex, the bilateral temporal pole, and left temporo-parietal junction. Conversely, memory representational areas such as the fusiform gyrus for faces and the posterior parietal cortex for locations did not change their activity with stabilization. The changes in activation in the precuneus, which also showed increased connectivity with the fusiform area, are likely to be related to the spatial nature of our task. The activation increase in the ventromedial prefrontal cortex, on the other hand, might reflect a general function in stabilized memory retrieval. This area might succeed the hippocampus in linking distributed neocortical representations.
\end{abstract}

When memorizing new information, distributing multiple shorter learning sessions over time is more efficient than one massed learning session (Carpenter and DeLosh 2006; Cepeda et al. 2006). The phenomenon that spaced learning leads to more stable memory traces than massed learning was confirmed experimentally in the late 1800's (Ebbinghaus 1885). This difference in the stability of memory traces can be related to two mechanisms: one affecting memory encoding at study and one affecting memory consolidation. Repeated processing of the same information in short succession leads to reduced neural activity in brain areas critically involved in memory encoding, like the left inferior prefrontal cortex (Wagner et al. 2000) or rhinal cortex (Fernández and Tendolkar 2006), which could explain the poorer performance at the time of retrieval. On the contrary, the time between encoding sessions in spaced learning allows the memory trace to consolidate into more stable forms that are more resistant to interference. This consolidation is particularly enhanced if the learning sessions are interceded by sleep (Ellenbogen et al. 2006). This consolidation process is dependent on specific stages of sleep (Stickgold 2005; Marshall et al. 2006; Rasch et al. 2007) and may include processes ranging from changes in local neural connectivity to system-level reorganization (McGaugh 2000). Our aim is to maximize the difference in trace stabilization by making use of these factors. One factor is the longer study-test interval to increase the time for consolidation. The other is the spaced learning scheme, which reduces interference and/or repetition suppression (Wagner et al. 2000; Fernández and Tendolkar 2006).

There is little information on how memory-trace stabilization is reflected in system-level network properties, especially in

${ }^{3}$ Corresponding author.

E-mail atsuko.takashima@fcdonders.ru.nl; fax 31-24-3610989.

Article is online at http://www.learnmem.org/cgi/doi/10.1101//m.605607. humans. The stabilization could be explained by the enhancement of local representations that are already active during memory retrieval of the newly learned memories. This would predict an increase in the activation of regions already engaged during the retrieval of not-yet stabilized (labile) associations. Alternatively, trace stabilization might be associated with largescale network reorganization (Frankland and Bontempi 2005). This would predict that brain areas that are not involved in retrieval of not-yet stabilized memory traces become engaged in retrieval of stabilized memories. To address this question, we performed a study comparing brain activity that was related to the retrieval of newly learned memory traces trained in a massed scheme (labile condition) with a spaced learning scheme (stabilized condition).

For the two conditions, subjects memorized sets of arbitrary face-location associations (Fig. 1A). We chose these particular stimuli because faces and locations are represented in distinctive and rather specific brain regions-an area in the inferior temporal cortex centered in the fusiform gyrus for faces (Kanwisher et al. 1997; Tsao et al. 2006) and the posterior parietal cortex for spatial locations (Sereno et al. 2001; Medendorp et al. 2005). After the training of these associations, brain activity was measured during a cued-recall test probing with the faces from the labile and the stabilized conditions randomly intermixed. Using event-related functional magnetic resonance imaging (fMRI), we set out to investigate whether stabilization of the memory traces is reflected as changes in retrieval-related activity in the representational brain regions of face and location (fusiform gyrus and posterior parietal cortex) or rather network-level reorganization engaging new brain regions. With respect to regions involved in network-level reorganization, there are several candidates. One candidate is the precuneus, which recently has received increased attention in studies on visuo-spatial representations and episodic memory (Cavanna and Trimble 2006). This area might be impor- 
A. Experimental scheme

\begin{tabular}{|c||c||c|}
\hline Day 1 & Day 4 & Day 7 \\
Training & Training & Training \\
Stabilized & Stabilized & Stabilized \\
6x passive $(p)$ & $4 \times(p)$ & $2 \times(p)$ \\
3x active $(a)$ & $12 \times(a)$ & $12 \times(a)$ \\
& & \\
Set A (20 pairs) & Set A \& B & Set A \& B \\
Set B (20 pairs) & & \\
\hline
\end{tabular}

B. Training scheme Training passive
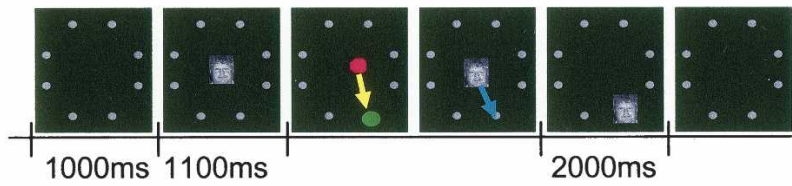

next trial

Training active (correct response)
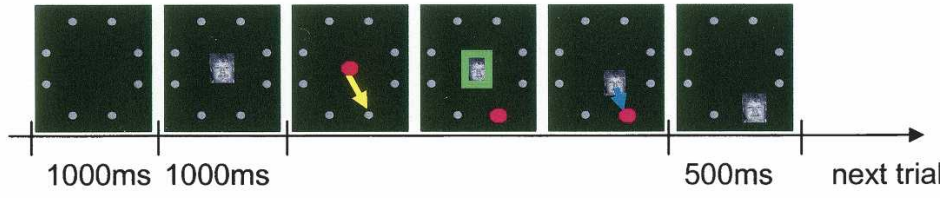

Training active (incorrect response)
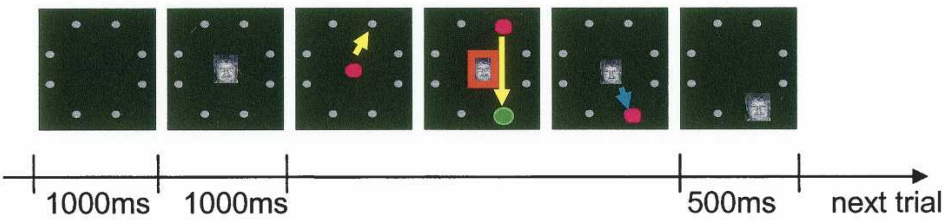

C. Recall test phase in the scanner

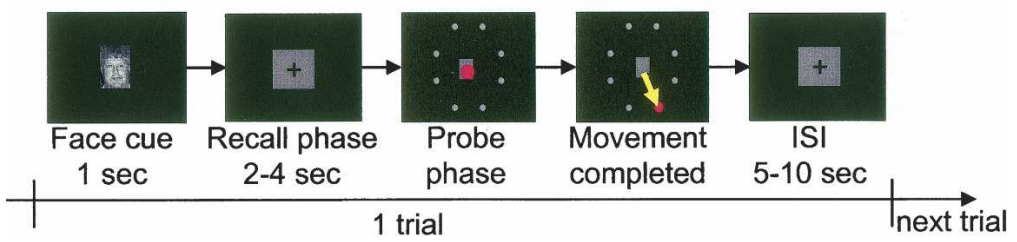

Figure 1. Experimental set-up. (A) Experimental scheme. Subjects were trained on the stabilized associations on three different days and trained on labile associations on the day of the recall test (day 8). (B) Training scheme. For the passive training, the location was cued as the green dot. After the subject moved the pink cursor to the location (yellow arrow) the face reappeared in the center and moved to the location (blue arrow) before disappearing after $2000 \mathrm{msec}$. In the active training, the subjects first had to move to a location when the face cue was presented (yellow arrow). Subsequently, a positive (green square) or a negative (red square) feedback was given. In case of a miss, the feedback was followed by the correct location (green circle) to which the subject then had to make a movement. This was followed by the face moving to the correct location and staying there for $500 \mathrm{msec}$. (C) Scheme during fMRI session. Here the subjects did not get any feedback.

tant in functioning as the connecting node between face and location representations, and its engagement might increase with trace stabilization. A second candidate for an area involved in network reorganization is the ventromedial prefrontal cortex (Bontempi et al. 1999; Takehara et al. 2003; Frankland and Bon- tempi 2005). In an earlier study, we demonstrated that retrieval-related brain activity shifts from the hippocampus to the ventromedial prefrontal cortex with memory trace stabilization (Takashima et al. 2006). We interpreted this as the hippocampus initially binding the multiple features of the memory trace, but with consolidation, the ventromedial prefrontal cortex took over the role as a binding node. However, this interpretation was partly confounded with reduced performance of the remote (consolidated) memory trace, leaving open the possibility that increased difficulty could explain the increase in ventromedial prefrontal cortex activation (McKiernan et al. 2003, 2006; Ridderinkhof et al. 2004). Here, we hypothesize that if we can observe an increase in the ventromedial prefrontal cortex activity with trace stabilization when performances for stabilized and labile associations are equal, then this will support the interpretation that this region takes over some of the hippocampal functions after the memory trace has been stabilized, independent of performance or task difficulty.

\section{Results}

This experiment was set up as a joint magnetoencephalography (MEG)-fMRI study. The subjects were trained on a paired associate task and brain activity during cued recall was subsequently recorded using both MEG and fMRI. The MEG results will be reported elsewhere. Twenty-two subjects were trained on two sets of face-location associations. The task of the subjects was to retrieve the associated location when a particular face cue was presented. One set was trained over the course of a week, with the three training sessions spaced over $3 \mathrm{~d}$ (stabilized condition), and the other set was learned on the day of measurement in one massed session (labile condition), but with the same amount of repetitions (39 times per pair) (Fig. $1 A, B)$. The subjects were measured with MEG and fMRI consecutively while they performed the cued-recall tests (for fMRI; Fig. 1C). Given that delayed responses were required in the MEG and MRI sessions, we included an extra behavioral task in order to characterize reaction times (see details in the Materials and Method section).
Stabilized associations are retrieved faster

During the behavioral task, reaction times were shorter in the stabilized condition than in the labile condition (mean $\pm \mathrm{SE}$ : stabilized $=936 \pm 26.1 \mathrm{msec}$, labile $=1029 \pm 35.0 \mathrm{msec} ; n=22$, $t=4.520, P<0.001$; Fig. $2 \mathrm{~A}$ ). Due to the repeated training, the 

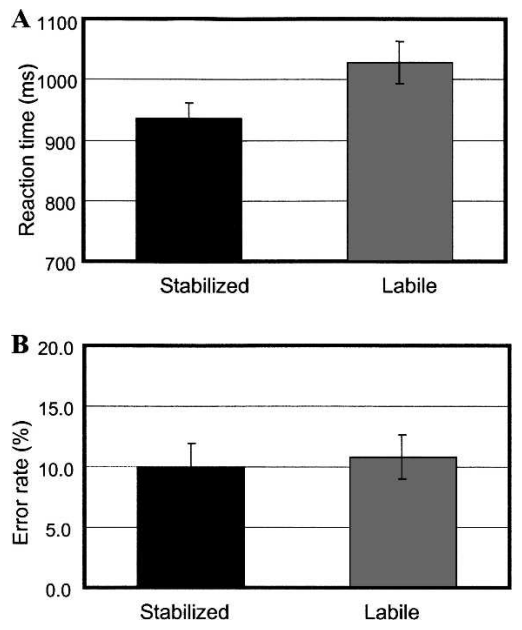

Figure 2. Behavioral results. Mean and standard error of the reaction time for stabilized (black) and labile (gray) conditions during the behavioral task $(A)$ and the error rate during the fMRI session $(B)$. Reaction times were significantly shorter for the stabilized condition $(P<0.05)$. Performance level did not differ between the stabilized and labile condition.

overall hit rates during the fMRI session were high and virtually identical for the labile and the stabilized conditions (mean $\pm \mathrm{SE}$ : stabilized $=90.1 \pm 2.0 \%$, labile $=89.2 \pm 1.8 \% ; t=0.754$, $P=0.46$; Fig. 2B). These behavioral results show that stabilized associations were retrieved faster but equally well, as compared to labile associations. This suggests a behavioral advantage of trace stabilization.

\section{Imaging results}

\section{Additional brain areas became engaged with memory stabilization}

To identify brain regions that show differential activity between stabilized and labile memory retrieval, we directly contrasted brain activity associated with trials related to successful memory retrieval of stabilized and labile associations. This contrast revealed that the successful retrieval of stabilized associations is accompanied by increased brain activity in midline structures, including the precuneus and the ventromedial prefrontal cortex. At the convexity, this contrast identified the left temporoparietal junction and the anterior aspect of the middle/superior temporal gyrus bilaterally (Table 1A; Fig. 3A). In contrast, we did not find significantly stronger effects for the retrieval of labile compared with stabilized associations. For both contrasts, no differences were detected in the areas typically associated with face and location representations (fusiform gyrus and posterior parietal cortex, respectively). Thus, our results suggest that a set of additional brain areas became engaged during memory retrieval of stabilized associations. This indicates large-scale network-level reorganization with trace stabilization rather than strengthening of the representational areas.

To identify changes in functional connectivity associated with trace stabilization, we performed a psycho-physiological interaction (PPI) analysis (Friston et al. 1997). Using the fusiform area as the seed region, this revealed significantly stronger correlated activity for the stabilized compared with the labile associations in the medial part of the precuneus bilaterally $(P=0.031$, corrected). The seed region was identified in individual subjects by contrasting the nonassociated face condition to the scrambled condition. This finding suggests that trace stabilization is associated with a stronger coupling of the fusiform gyrus and the precuneus during memory retrieval (Fig. 3B).
Overlap between memory retrieval of stabilized and labile associations

To identify those brain areas that were commonly activated during the retrieval of stabilized and labile associations, we searched for overlapping regions for the stabilized versus non-memory contrast and the labile versus non-memory contrast. This analysis revealed areas in the posterior parietal cortex bilaterally, with the left cluster extending to the precentral gyrus and superior frontal gyrus. In addition, we observed activations in the right fusiform gyrus, the right posterior precuneus extending to the retrosplenial cortex, the left middle occipital gyrus, and the left basal ganglia, with a cluster centered in the putamen-pallidum complex (Table 1B; Fig. 3C).

\section{Hemifield-specific spatial representations in posterior parietal cortex}

Furthermore, we tested whether the posterior parietal cortex activations, observed here in the retrieval of long-term memory items, are associated with hemifield-specific spatial representations found in working memory paradigms (Sereno et al. 2001; Medendorp et al. 2005). For this reason, we chose the retinotopic hemifield-related region of interest in the posterior parietal cortex, as observed in a previous study, where subjects had to retain the spatial location during a working memory task (Medendorp et al. 2005). The two spherical regions of interest (radius, $20 \mathrm{~mm}$ ) were centered at the local maxima of that study [-26 -66 46], [20 - 66 54], in the left and right hemisphere, respectively. Indeed, we found relatively stronger activation in the left posterior parietal cortex when subjects were retrieving a location on the right hemifield $\left(\mathrm{P}_{\mathrm{SVC}}=0.001\right.$, small volume corrected; $\left.\mathrm{SVC}\right)$ and in the right posterior parietal cortex for the left hemifield $\left(\mathrm{P}_{\mathrm{SVC}}=0.002\right.$; Fig. 3D). This finding suggests that brain activation associated with hemifield-specific spatial representations in working memory tasks is also engaged during long-term memory retrieval. The parameter estimates (beta weights) of all four experimental conditions (stabilized, labile, nonassociated, and scrambled) contrasted with explicit baseline (intertrial interval) in the two local maxima of this hemifield-specific effect (left posterior parietal cortex [-18 - 7646$]$ and right posterior parietal cortex [20 - 60 54]; Fig. 2D) suggest that these brain regions are involved in spatial memory retrieval independent of trace stabilization. Thus, contralateral posterior parietal cortex activation was observed for retrieval of hemifield-specific information on spatial locations, but the strength of this activation did not change with the stabilization of the underlying memory trace.

\section{Discussion}

Here, we have shown that memory stabilization is associated with faster access to stored traces, confirming the behavioral advantage of trace stabilization (Ebbinghaus 1885). The task used in this study required the subjects to recollect the location when cued with a face. Consequently, we identified a hemifieldspecific area in the posterior parietal cortex related to the spatial memory trace. However, this representational area did not change its retrieval activity strength with trace stabilization. On the contrary, retrieval of a stabilized memory in contrast to a labile one involved changes in neural activity in the precuneus, the ventromedial prefrontal cortex, the bilateral temporal poles, and the left temporo-parietal junction. This suggests a large-scale network-level reorganization with stabilization. Furthermore, a psycho-physiological interaction analysis revealed a strengthening in the functional connectivity between the fusiform and the precuneus with trace stabilization.

Recently, the precuneus has come into focus (for review, see Cavanna and Trimble 2006) and has been shown to be involved in a wide spectrum of highly integrated tasks, including visuospatial imagery, episodic memory retrieval, and self-processing 
Table 1. Functional MRI results

\section{A. The stabilized contrasted to the labile condition}

\begin{tabular}{|c|c|c|c|c|c|}
\hline \multirow[b]{2}{*}{ Brain region } & \multirow[b]{2}{*}{ Cluster size } & \multirow[b]{2}{*}{ Cluster P } & \multicolumn{3}{|c|}{ Local maxima MNI coordinates } \\
\hline & & & $x$ & $y$ & $z$ \\
\hline $\begin{array}{l}\text { Right medial frontal gyrus_-BA10 } \\
\text { Right ventromedial prefrontal cortex-BA32 } \\
\text { Left medial frontal gyrus-BA10 } \\
\text { Left ventromedial prefrontal cortex-BA32 }\end{array}$ & $\begin{array}{l}252 \\
252 \\
252 \\
252\end{array}$ & $\begin{array}{l}0.013 \\
0.013 \\
0.013 \\
0.013\end{array}$ & $\begin{array}{r}6 \\
2 \\
-4 \\
-12\end{array}$ & $\begin{array}{l}52 \\
48 \\
52 \\
48\end{array}$ & $\begin{array}{r}8 \\
2 \\
6 \\
-4\end{array}$ \\
\hline $\begin{array}{l}\text { Left precuneus } \\
\text { Right precuneus }\end{array}$ & $\begin{array}{l}515 \\
515\end{array}$ & $\begin{array}{l}<0.001 \\
<0.001\end{array}$ & $\begin{array}{r}-4 \\
2\end{array}$ & $\begin{array}{l}-54 \\
-50\end{array}$ & $\begin{array}{l}36 \\
34\end{array}$ \\
\hline $\begin{array}{l}\text { Left inferior parietal lobule-BA40 } \\
\text { Left inferior parietal lobule-BA40 } \\
\text { Left supramarginal gyrus } \\
\text { Left angular gyrus }\end{array}$ & $\begin{array}{l}967 \\
967 \\
967 \\
967\end{array}$ & $\begin{array}{l}<0.001 \\
<0.001 \\
<0.001 \\
<0.001\end{array}$ & $\begin{array}{r}-58 \\
-54 \\
-4 \\
-46\end{array}$ & $\begin{array}{l}-36 \\
-36 \\
-42 \\
-54\end{array}$ & $\begin{array}{l}26 \\
28 \\
30 \\
28\end{array}$ \\
\hline $\begin{array}{l}\text { Left superior temporal gyrus_-BA38 } \\
\text { Left middle temporal gyrus }\end{array}$ & $\begin{array}{l}185 \\
185\end{array}$ & $\begin{array}{l}0.045 \\
0.045\end{array}$ & $\begin{array}{l}-52 \\
-50\end{array}$ & $\begin{array}{l}12 \\
14\end{array}$ & $\begin{array}{l}-22 \\
-32\end{array}$ \\
\hline $\begin{array}{l}\text { Right middle temporal gyrus_-BA21 } \\
\text { Right middle temporal gyrus_-BA38 }\end{array}$ & $\begin{array}{l}502 \\
502\end{array}$ & $\begin{array}{l}<0.001 \\
<0.001\end{array}$ & $\begin{array}{l}60 \\
40\end{array}$ & $\begin{array}{l}4 \\
8\end{array}$ & $\begin{array}{l}-18 \\
-42\end{array}$ \\
\hline
\end{tabular}

B. Overlap of the stabilized and the labile contrast to non-memory condition

\begin{tabular}{|c|c|c|c|c|c|}
\hline \multirow[b]{2}{*}{ Brain region } & \multirow[b]{2}{*}{ Cluster size } & \multirow[b]{2}{*}{ Cluster P } & \multicolumn{3}{|c|}{ Local maxima $\mathrm{MNI}$ coordinates } \\
\hline & & & $x$ & $y$ & $z$ \\
\hline $\begin{array}{l}\text { Left posterior parietal lobule-BA7 } \\
\text { Left inferior parietal lobule-BA40 } \\
\text { Left postcentral gyrus-BA3 } \\
\text { Left postcentral gyrus-BA40 } \\
\text { Left precentral gyrus-BA4 } \\
\text { Left middle frontal gyrus-BA6 }\end{array}$ & $\begin{array}{l}2303 \\
2303 \\
2303 \\
2303 \\
2303 \\
2303\end{array}$ & $\begin{array}{l}<0.001 \\
<0.001 \\
<0.001 \\
<0.001 \\
<0.001 \\
<0.001\end{array}$ & $\begin{array}{l}-20 \\
-38 \\
-40 \\
-38 \\
-40 \\
-28\end{array}$ & $\begin{array}{l}-60 \\
-46 \\
-26 \\
-30 \\
-16 \\
-10\end{array}$ & $\begin{array}{l}54 \\
56 \\
54 \\
46 \\
60 \\
58\end{array}$ \\
\hline $\begin{array}{l}\text { Right posterior parietal lobule } \\
\text { Right precuneus }\end{array}$ & $\begin{array}{l}204 \\
204\end{array}$ & $\begin{array}{l}<0.001 \\
<0.001\end{array}$ & $\begin{array}{l}16 \\
12\end{array}$ & $\begin{array}{l}-66 \\
-68\end{array}$ & $\begin{array}{l}54 \\
67\end{array}$ \\
\hline Right fusiform gyrus & 177 & 0.003 & 34 & -48 & -22 \\
\hline Left middle occipital gyrus & 112 & 0.014 & -26 & -76 & 32 \\
\hline $\begin{array}{l}\text { Right retrosplenial cortex } \\
\text { Right precuneus }\end{array}$ & $\begin{array}{l}204 \\
204\end{array}$ & $\begin{array}{l}0.002 \\
0.002\end{array}$ & $\begin{array}{l}20 \\
20\end{array}$ & $\begin{array}{l}-60 \\
-64\end{array}$ & $\begin{array}{l}28 \\
30\end{array}$ \\
\hline $\begin{array}{l}\text { Left putamen } \\
\text { Left putamen } \\
\text { Left globus pallidus } \\
\text { Left globus pallidus }\end{array}$ & $\begin{array}{l}525 \\
525 \\
525 \\
525\end{array}$ & $\begin{array}{l}<0.001 \\
<0.001 \\
<0.001 \\
<0.001\end{array}$ & $\begin{array}{l}-16 \\
-18 \\
-24 \\
-20\end{array}$ & $\begin{array}{r}14 \\
18 \\
-18 \\
-6\end{array}$ & $\begin{array}{r}-8 \\
-2 \\
0 \\
10\end{array}$ \\
\hline
\end{tabular}

operations (e.g., judgment on one's own versus another person's face/personality traits). For instance, Frings et al. (2006) showed a bilateral posterior precuneus activation during both encoding and recognition of allocentric, viewpoint-independent spatial memory. In a working memory paradigm, Wallentin et al. (2006) also showed bilateral precuneus activity for the recall of spatial information contrasted to recall of nonspatial information. Since the precuneus has been implicated in episodic memory retrieval and visuospatial attention, it is likely that this area becomes more engaged with the stabilization of the memory trace for the association of a face with a location. The result of the functional connectivity analysis also supports the notion that the correlation between the fusiform area, which is activated during face perception, and the precuneus, strengthens with trace stabilization. The extent of the anatomical connectivity of the precuneus is widespread and involves higher associative cortical and subcortical structures (for review, see Cavanna and Trimble 2006). It has direct connections with other parietal areas including the posterior parietal cortex (Selemon and Goldman-Rakic 1988; Cavada and Goldman-Rakic 1989; Leichnetz 2001), an area identified as location-representation storage area in this study. The precuneus also has connections to other brain regions, including the posterior cingulate cortex (Leichnetz 2001), which, in turn, connects to the medial temporal lobe and the inferior temporal cortex, including the fusiform gyrus. Thus, from an anatomical point of view, this area is optimally interconnected with a wide range of relevant brain areas for processing higher order associations in multimodal and, in particular, spatial tasks. Even though there might be no direct anatomical connection between the fusiform gyrus and the precuneus, it is highly probable that these two areas are functionally coupled and indirectly connected during retrieval of face-location associations.

Another difference in brain activity between the stabilized and labile conditions was found in the ventromedial prefrontal cortex. The effect appears to be in line with our previous finding (Takashima et al. 2006), where the ventromedial prefrontal activation strengthened with the remoteness of the retrieved memory. What is the function of the ventromedial prefrontal region in retrieving stabilized memories? Based on converging evidence from activation studies in rodents and humans, as well as lesion studies in animals (Bontempi et al. 1999; Takehara et al. 2003; Frankland et al. 2004; Teixeira et al. 2006), it has been hypothesized that, after consolidation or trace stabilization, medial prefrontal cortex takes over the hippocampal role as a link- 

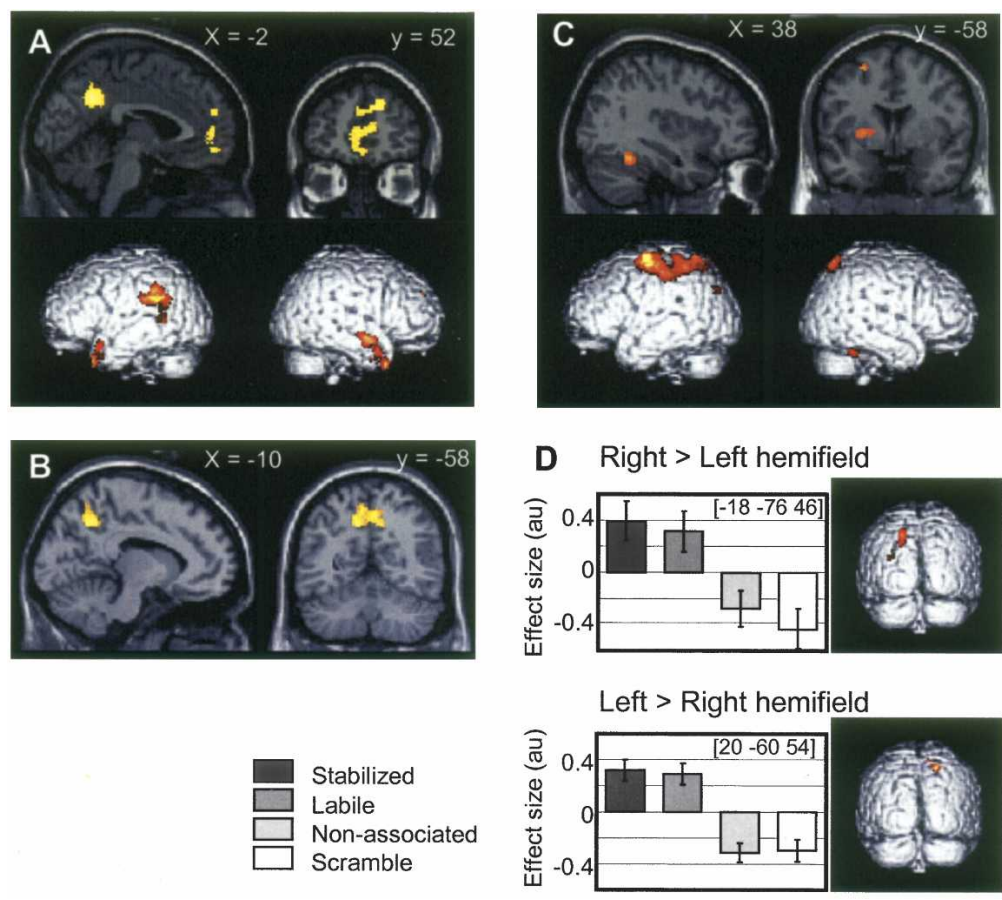

Figure 3. $f M R I$ results. Effects are depicted on selected slices of a high-resolution $T 1$ image or 3D renderings provided by SPM2 applying a cluster threshold of $P<0.05$, corrected. ( $A$ ) Effects found for the stabilized versus the labile memory condition. The sagittal slice shows both the precuneus and the ventromedial prefrontal effect. (B) Functional connectivity analysis showed a positive correlation between activity in the seed region in the fusiform area and the precuneus, which increased with trace stabilization. (C) Conjunction analysis showing the overlap of activations found for stabilized versus the non-memory contrast and the labile versus the non-memory contrast. The sagittal slice shows a right fusiform cluster of activated voxels and the coronal slice shows an effect in the left basal ganglia. $(D)$ Hemifield-specific activation related to location representations was found in the contralateral posterior parietal cortex (PPC). The bar graphs show the mean and $90 \%$ confidence interval of the effect size (au) for the local maxima of the PPC effect for the four conditions (stabilized, labile, non-memory association, and scramble) relative to the explicit baseline (intertrial interval).

ing node for spatially distributed neocortical sites. Such a putative linking node is supposed to associate distributed neocortical representations into a coherent memory trace (Frankland and Bontempi 2005). Most previous findings might have been confounded by differences in task difficulty or performance differences with poorer memory for remote (stabilized) as compared with recent (labile) conditions. Rudy et al. (2005) have suggested that old or remote memories are weaker than recent ones, and therefore require more prefrontally dependent control processes in order to reactivate them. In our study, however, we explicitly trained the subjects in such a way that retrieval performances for stabilized and labile conditions were similar. Nevertheless, and contradictory to Rudy's theory, we identified a ventromedial prefrontal effect in the current study, despite the fact that the retrieval accuracy of the stabilized association was virtually identical and the retrieval speed was even faster compared with the labile condition. Thus, this effect cannot be explained by increased task difficulty (McKiernan et al. 2003, 2006; Ridderinkhof et al. 2004) or poorer performance. We conclude that the ventromedial prefrontal cortex may indeed take over a higher order integrative function for stabilized memory traces. Nevertheless, it is still conceivable that, since information in the stabilized condition had multiple contexts from three different encoding episodes, this factor might have caused an activation increase in the ventromedial prefrontal cortex, reflecting activity associated with retrieval of conflicting temporal contexts. However, the location of our effect does not overlap with activations found in studies probing conflicting information, which are found more dorsally.

Which factors might account for the changes in brain activation with stabilization of the memory trace? In studies focusing on memory consolidation, forgetting with the passing of time is a confound that is difficult to control. To address the change in brain activity due to trace stabilization, we chose the strategy of training people so well that the recall performances were comparable for the stabilized and labile conditions. Using this strategy, several aspects were involved in stabilizing a memory trace. One important element was the difference in the number of training sessions. Even though the conditions were the same with respect to the number of repetitions, the trainings for the stabilized condition were done on three different days, whereas training for the labile condition was done in $1 \mathrm{~d}$. This approach could have provided more rich contextual information for the stabilized than labile condition. This additional contextual information might come into play at the time of retrieval and could be reflected in the activation of the engaged brain regions. Another element was the time between each encoding session and recall test. Longer time intervals have been proposed to result in progression of consolidation, in particular during sleep. The more consolidated the memory trace becomes the less it is prone to interference (Walker et al. 2003). Thus, for the stabilized condition, the periods between the study sessions are likely to allow for consolidation of the memory trace. Finally, the differences in intervals between repeated learning have consequences for repetition suppression. If new material with elements in common is studied in short successions, repetition suppression will reduce the stimulus-specific activation, which might be important for encoding (Wagner et al. 2000; Fernández and Tendolkar 2006). Since the labile condition was studied in a massed style compared with the stabilized condition, this could have reduced the brain activity during the study, and subsequently resulted in differences in activity at the time of retrieval. Future studies investigating these factors are needed to account for their individual contributions to the stabilization of the memory trace and the brain activity involved.

In addition to the precuneus and the ventromedial prefrontal cortex, we found greater activation for the stabilized condition relative to the labile condition in the temporal pole bilaterally and the left inferior parietal cortices close to the temporoparietal junction. Anterior temporal cortex activation is often associated with autobiographical memory tasks (Fink et al. 1996; Piefke et al. 2003), although it has also been found during memory retrieval of general facts and events (Maguire et al. 2000). In a recent study investigating the activation of personal or famous names to unfamiliar names, Sugiura et al. (2006) reported increased activity in the bilateral temporal poles and anterolateral temporal cortices, as well as the left temporo-parietal junction for personally known or famous names compared with unfamiliar names. This set of areas is similar to that found in our 
stabilized to labile condition contrast. In the debriefing after the experiment, many of our subjects expressed that they tried to associate the faces to someone they already knew, such as family members and friends, in order to perform the task better. In this regard, we could speculate that the memory traces of the faces that were embedded into an autobiographical memory network were the better-stabilized traces.

So far, not many studies have investigated long-term memory representations for spatial location (e.g., Burgess et al. 2001; Düzel et al. 2003; Khader et al. 2005). To our knowledge, no attempt has been made to probe long-term memory representations of space or locations that are hemifield specific. The parietal areas activated during a working memory task of hemifieldspecific locations (Medendorp et al. 2005) were also activated during our task, when subjects recollected associated locations from long-term memory. This activation could be interpreted as either associated with the representation of the location or with the movement preparation to the assigned location. Yet, this area is known to be activated even when subjects are not instructed to move to the location on the screen after a retention period in a delayed-match-to-sample task (Sereno et al. 2001). Thus, it is more likely that the effect found here is related to recollection and maintenance of visuo-spatial information rather than motor preparation. Our result suggests that spatial representations maintained during working memory tasks are also used during long-term memory retrieval and that the hemifield-specific representation is located in the contralateral posterior parietal cortex.

The hippocampus is thought to be critical for spatial associative processing (e.g., Davachi and Wagner 2002; Sommer et al. 2005; Piekema et al. 2006), and it is also activated at the time of retrieval (Düzel et al. 2003). The classical consolidation theory proposes that the hippocampal contribution to memory retrieval vanishes with trace stabilization (Alvarez and Squire 1994). Orban et al. (2006) have shown that for spatial memory, a night of sleep is necessary for the trace to transfer from the hippocampus to the basal ganglia. In contrast, we did not find evidence for hippocampal participation even during memory retrieval of the labile condition, which was tested before the subjects had time to sleep. One might ask why we did not find activity in the hippocampus. Probably, the massive training scheme of 39 repetitions per association as applied here caused establishment of stimulus-response associations, which can be formed rather rapidly in the basal ganglia (Fig. 3C) and thus retrieved independent of the hippocampus (Packard 1999; Hartley et al. 2003; Iaria et al. 2003; Voermans et al. 2004). However, it is important to note that interpretation of null findings, especially when looking at hippocampal effects with fMRI, should be done with caution.

While previous studies on memory stabilization and consolidation have focused on the role of the hippocampus, we have addressed the role of neocortical brain structures. Even though we did not find changes in areas responsible for the actual representations, we did find changes in higher executive areas. The changes in the precuneus are likely to be related to the spatial specificity of the task, whereas the changes in the ventromedial prefrontal cortex are more likely to be independent of the modality of the memory items. Interpreted in conjunction with the previous study (Takashima et al. 2006) and several animal studies (Bontempi et al. 1999; Takehara et al. 2003), the ventromedial prefrontal cortex is likely to take over the role of the hippocampus, linking distributed representations as a consequence of memory consolidation. Future studies are required to further elucidate the exact role of the ventromedial prefrontal cortex in orchestrating the retrieval of stabilized memory representations.

\section{Materials and Methods}

\section{Subjects}

Twenty-two right-handed subjects participated (11 females, 19$39 \mathrm{yr}$ of age range; average $23.3 \pm 4.8 \mathrm{yr}$ ). Participants received either a monetary fee or study credits for their participation. None of the subjects used medication or had a history of drug abuse, head trauma, or neurological/psychiatric illness. Written informed consent was obtained according to the local medical ethics committee. One subject could not undergo fMRI scanning due to claustrophobia and was omitted from the MRI data analyses.

\section{Stimuli}

One hundred grayscale front-facing photographs of unfamiliar male and female faces (50 of each) were used as face stimuli. The face stimuli were of anonymous people visiting a museum, taken with a digital camera by one of the investigators (I.N.). The pictures were comprised of the neck and above, all showing neutral expressions gazing toward the camera. These faces were divided into five sets of 20 faces each (10 male and 10 female) and were assigned the following conditions: Stabilized for MEG and fMRI sessions (set A), stabilized for fMRI session only (set B), labile for MEG and fMRI sessions (set C), labile for fMRI session only (set D), and nonassociated sets (set E) (Fig. 3A). The assignment of each stimulus to each condition was counter balanced among subjects. In addition, we constructed 20 scrambled faces as nonfacial perception stimuli by spatially Fourier transforming 15 face stimuli and shuffling the phase to construct scrambled images that were equal in luminance, spatial frequency, and contrast to the original face stimuli. For the low-level baseline, a black fixation cross in a gray box was presented during the intertrial interval.

\section{Procedure}

\section{Experimental task}

Subjects were trained to associate each of the 80 faces that were presented in the center of the computer screen to one of the eight locations that were surrounding the center with equal distance (Fig. 1B). For both the labile and stabilized condition, we combined two types of training, so-called passive and active training. In the "passive training," a face appeared in the center of the screen surrounded by eight gray circles for $1100 \mathrm{msec}$. This was followed by a color change of the target location from gray to green, thus prompting the subjects to make a joystick movement to the cued location. After the cursor reached the correct location, the face reappeared in the center and moved to the location and stayed there for 2000 msec. In the "active training," the target location was not cued, and the subjects had to recall the associated location from memory when the face appeared (face disappeared after $1000 \mathrm{msec}$, regardless of the speed of the subject's response). If the correct location was chosen, a green box appeared in the middle and the face moved to that location, where it stayed for $500 \mathrm{msec}$. If an incorrect location was chosen, feedback was given by showing a red box and the correct target location. Subsequently, the subject had to make a movement to the correct location, after which the face moved to that location where it stayed for $500 \mathrm{msec}$ before the next trial started. "Passive training" was used mainly in the beginning of the training; at the end, mainly "active training" was used (see below for details).

\section{Experimental procedure on days 1-7}

For the stabilized condition, subjects were trained to associate 40 different faces (sets A and B) to one of the eight locations in three different training days. On day 1 , subjects were trained with nine repetitions (six times passive and three times active) for each of the pairs. On the second training day, which took place between day 3 and day 5 , the subjects were trained again with the same 40 pairs, but with 16 repetitions for each pair (four times passive, 12 times active). The third training day took place on day 7 with 14 
repetitions (two times passive, 12 times active). In total, the subjects were exposed to 39 repetitions of each face-location pair.

\section{Experimental procedure on day 8}

\section{Training set C (labile condition)}

On the morning of day 8 , subjects were trained on a new set of 20 face-location associations, applying exactly the same scheme and the equal amount of training as the stabilized set (39 repetitions, 12 times passive, 27 times active), except that it was massed.

\section{Familiarization of set $E$ (nonassociated condition) and MEG measurement}

Additionally, subjects were familiarized with another set of 20 faces (nonassociated face stimuli; set E) just prior to the MEG session by passively observing the faces that were shown in the center of the computer screen consecutively for 2 sec each with 15 repetitions. We performed this familiarization task to avoid the confounding effect of novelty detection. This was followed by an MEG session, where subjects were tested on the cued recall of 20 stabilized (set A) and 20 labile (set C) associations.

\section{Behavioral task}

In this test, each face stimulus of sets $\mathrm{A}$ and $\mathrm{C}$ appeared sequentially in a randomized order in the center of the screen, and subjects were asked to move the cursor with the joystick to the associated location as quickly and accurately as possible.

\section{Training set $D$ (labile condition)}

After a lunch break, subjects learned another set of 20 new facelocation pairs in the same manner as in set C.

\section{Cued-recall test (fMRI)}

The training of set D was followed by the fMRI investigation. In the scanner, stimuli were projected onto a screen viewed by participants through a mirror mounted on the head radiofrequency coil while the subject was lying supine in the scanner. While scanning, subjects saw a sequential series of face stimuli (randomly from sets A-D, intermixed with 15 nonassociated face trials from set E) and 15 scramble trials, all of which were presented twice during the whole session. Each item was presented for $1 \mathrm{sec}$, followed by a delay period of $2-4 \mathrm{sec}$, during which a central fixation cross was presented. The fixation period was followed by a probe stimulus showing eight gray circles presented at the trained locations surrounding the central fixation cross. For the stabilized and labile conditions, the subject had to move the cursor to the associated location circle, and no feedback was given. For the nonassociated face and scramble trials, one of the gray circles appeared green in the probe phase and the subjects were instructed to move the cursor to that circle. An intertrial interval of 5-10 sec with a fixation cross appeared between each trial. Subjects were instructed to fixate continuously onto the central cross after the face cue was presented and wait for the joystick movement until the probe circles and the cursor appeared (Fig. 1C). Successful recall (hit) was defined by a correct response within 4 sec after probe onset.

\section{MRI data acquisition}

For fMRI we acquired, with ascending slice acquisition, a T2*weighted echo-planar imaging (EPI) sequence (Sonata $1.5 \mathrm{~T}$, Siemens; 33 axial slices, volume-repetition time $[\mathrm{TR}]=2.29 \mathrm{sec}$, echo time $[\mathrm{TE}]=30 \mathrm{msec}, 90^{\circ}$ flip-angle, matrix $=64 \times 64$, slice thickness $=3.0 \mathrm{~mm}$, slice gap $=0.5 \mathrm{~mm}$, field of view $[\mathrm{FOV}]=224 \mathrm{~mm})$. For structural MRI, we acquired a T1-weighted magnetization-prepared, rapid acquisition gradient echo (MPRAGE) sequence (240 coronal slices, volume-TR $=1660 \mathrm{msec}$, $\mathrm{TE}=2.02 \mathrm{msec}$, inversion time $=750 \mathrm{msec}, 8^{\circ}$ flip-angle, matrix $=256 \times 256$, slice thickness $=1.0 \mathrm{~mm}$, no slice gap, $\mathrm{FOV}=256 \mathrm{~mm})$.

\section{MRI data analysis}

Image pre-processing and statistical analysis was performed using the SPM2 software (http://www.fil.ion.ucl.ac.uk). The functional EPI-BOLD (blood-oxygenation level-dependent signal) images were realigned and the subject-mean was co-registered with the corresponding structural MRI using mutual information optimization. These were subsequently slice-time corrected, spatially normalized, and transformed into a common space, as defined by the SPM2 MNI T1 template, as well as spatially filtered by convolving the functional images with an isotropic 3D Gaussian kernel (10 mm full width at half maximum; FWHM). The fMRI data were analyzed statistically using the general linear model and statistical parametric mapping. Since sets A and B were trained in a spaced scheme, including nights of sleep to consolidate between training sessions, we collapsed these trials as the stabilized condition. Mass trained sets C and D were also collapsed as the labile condition to increase statistical power. The explanatory variables (stabilized hits, labile hits, nonassociation hits, scrambles hits, motor-response period, trials of no interest comprising of all missed trials and trials that took longer than 4 sec, and intertrial intervals) were temporally convolved with the canonical hemodynamic response function along with its temporal derivatives provided by SPM2. Regressor of the intertrial interval was used as the explicit baseline activation. The design matrix also included the realignment parameters to account for any residual movement-related effect. A high pass filter was implemented using a cut-off period of $128 \mathrm{sec}$ in order to remove the low-frequency effects from the time series. Nonassociated condition and scramble condition were collapsed as nonmemory condition when stabilized/labile to non-memory contrasts were tested. For the comparison of left and right hemifield location memory, we pooled data from stabilized and labile hits to increase statistical power and separated them subsequently according to their screen locations. Trials associated with the two most-left screen locations were contrasted with trials associated with the two most-right screen locations. For small volume correction (SVC), we chose two spherical regions of interest (radius, $20 \mathrm{~mm}$ ) centered at the local maxima of a previous study reporting retinotopic mapping of the posterior parietal region $[-26$ - 66 46], [20 - 66 54] (Medendorp et al. 2005). For the statistical analysis, relevant contrast parameter images were generated for each subject and subsequently subjected to a second-level random effects analysis with nonsphericity correction for correlated repeated measures where relevant. In the whole brain search, the results of the random effects analysis were thresholded initially at $P=0.001$ (uncorrected) and then the cluster-size statistics were used as the test statistic applying a threshold of $P \leq 0.05$ (corrected).

To probe changes in functional connectivity associated with stabilization, we performed a psychophysiological interaction (PPI) analysis embedded in SPM2. For this analysis, each subject's face area, a cluster of activation in the dominant hemisphere of the inferior-temporal cortex for the nonassociated face condition to scramble contrast, was used as the seed region. PPI regressors were calculated using this seed region for the stabilized versus the labile condition. The PPI regressors were inserted as userspecified regressors in new session-specific design matrices and the contrast for the PPI was calculated for each subject. Then, the subject-specific contrast images for the interaction were used as inputs for the second-level random effects analysis. At the second level, initially a one-sample $t$-test was conducted with a threshold of $P=0.01$ (uncorrected), and then the cluster-size statistics were used as the test statistic applying a threshold of $P \leq 0.05$ (corrected). This analysis was limited to the brain regions that were active $(P<0.05$ uncorrected) for the stabilized versus the labile condition.

\section{Acknowledgments}

This research was supported by grant from the Netherlands Organization for Scientific Research (NWO, 051.04.100).

\section{References}

Alvarez, P. and Squire, L.R. 1994. Memory consolidation and the medial temporal lobe: A simple network model. Proc. Natl. Acad. Sci. 91: 7041-7045.

Bontempi, B., Laurent-Demir, C., Destrade, C., and Jaffard, R. 1999. 
Time-dependent reorganization of brain circuitry underlying long-term memory storage. Nature 400: 671-675.

Burgess, N., Maguire, E.A., Spiers, H.J., and O'Keefe, J. 2001. A temporoparietal and prefrontal network for retrieving the spatial context of lifelike events. Neuroimage 14: 439-453.

Carpenter, S.K. and DeLosh, E.L. 2006. Impoverished cue support enhances subsequent retention: Support for the elaborative retrieval explanation of the testing effect. Mem. Cognit. 34: 268-276.

Cavada, C. and Goldman-Rakic, P.S. 1989. Posterior parietal cortex in rhesus monkey: II. Evidence for segregated corticocortical networks linking sensory and limbic areas with the frontal lobe. J. Comp. Neurol. 287: 422-445.

Cavanna, A.E. and Trimble, M.R. 2006. The precuneus: A review of its functional anatomy and behavioural correlates. Brain 129: 564-583.

Cepeda, N.J., Pashler, H., Vul, E., Wixted, J.T., and Rohrer, D. 2006. Distributed practice in verbal recall tasks: A review and quantitative synthesis. Psychol. Bull. 132: 354-380.

Davachi, L. and Wagner, A.D. 2002. Hippocampal contributions to episodic encoding: Insights from relational and item-based learning. J. Neurophysiol. 88: 982-990.

Düzel, E., Habib, R., Rotte, M., Guderian, S., Tulving, E., and Heinze, H.J. 2003. Human hippocampal and parahippocampal activity during visual associative recognition memory for spatial and nonspatial stimulus configurations. J. Neurosci. 23: 9439-9444.

Ebbinghaus, H. 1885. Über das Gedächtnis. Untersuchungen zur experimentellen Psychologie. Duncker \& Humblot, Leipzig, Germany. [The English edition is Ebbinghaus, H. (1913). Memory. A Contribution to experimental psychology. Teachers College, Columbia University, New York.]

Ellenbogen, J.M., Hulbert, J.C., Stickgold, R., Dinges, D.F., and Thompson-Schill, S.L. 2006. Interfering with theories of sleep and memory: Sleep, declarative memory, and associative interference. Curr. Biol. 16: 1290-1294.

Fernández, G. and Tendolkar, I. 2006. The rhinal cortex: 'Gatekeeper' of the declarative memory system. Trends Cogn. Sci. 10: 358-362.

Fink, G.R., Markowitsch, H.J., Reinkemeier, M., Bruckbauer, T., Kessler, J., and Heiss, W.-D. 1996. Cerebral representation of one's own past: Neural networks involved in autobiographical memory. J. Neurosci. 16: $4275-4282$.

Frankland, P.W. and Bontempi, B. 2005. The organization of recent and remote memories. Nat. Rev. Neurosci. 6: 119-130.

Frankland, P.W., Bontempi, B., Talton, L.E., Kaczmarek, L., and Silva, A.J. 2004. The involvement of the anterior cingulate cortex in remote contextual fear memory. Science 304: 881-883.

Frings, L., Wagner, K., Quiske, A., Schwarzwald, R., Spreer, J., Halsband, U., and Schulze-Bonhage, A. 2006. Precuneus is involved in allocentric spatial location encoding and recognition. Exp. Brain Res. 173: 661-672.

Friston, K.J., Buechel, C., Fink, G.R., Morris, J., Rolls, E., and Dolan, R.J. 1997. Psychophysiological and modulatory interactions in neuroimaging. Neuroimage 6: 218-229.

Hartley, T., Maguire, E.A., Spiers, H.J., and Burgess, N. 2003. The well-worn route and the path less traveled: Distinct neural bases of route following and wayfinding in humans. Neuron 37: 877-888.

Iaria, G., Petrides, M., Dagher, A., Pike, B., and Bohbot, V.D. 2003. Cognitive strategies dependent on the hippocampus and caudate nucleus in human navigation: Variability and change with practice. J. Neurosci. 23: 5945-5952.

Kanwisher, N., McDermott, J., and Chun, M.M. 1997. The fusiform face area: A module in human extrastriate cortex specialized for face perception. J. Neurosci. 17: 4302-4311.

Khader, P., Burke, M., Bien, S., Ranganath, C., and Rosler, F. 2005. Content-specific activation during associative long-term memory retrieval. Neuroimage 27: 805-816.

Leichnetz, G.R. 2001. Connections of the medial posterior parietal cortex (area 7m) in the monkey. Anat. Rec. 263: 215-236.

Maguire, E.A., Mummery, C.J., and Buchel, C. 2000. Patterns of hippocampal-cortical interaction dissociate temporal lobe memory subsystems. Hippocampus 10: 475-482.

Marshall, L., Helgadottir, H., Molle, M., and Born, J. 2006. Boosting slow oscillations during sleep potentiates memory. Nature 444: 610-613.

McGaugh, J.L. 2000. Memory-A century of consolidation. Science 287: 248-251.

McKiernan, K.A., Kaufman, J.N., Kucera-Thompson, J., and Binder, J.R. 2003. A parametric manipulation of factors affecting task-induced deactivation in functional neuroimaging. J. Cogn. Neurosci. 15: $394-408$.
McKiernan, K.A., D'Angelo, B.R., Kaufman, J.N., and Binder, J.R. 2006. Interrupting the "stream of consciousness": An fMRI investigation. Neuroimage 29: 1185-1191.

Medendorp, W.P., Goltzm, H.C., and Vilis, T. 2005. Remapping the remembered target location for anti-saccades in human posterior parietal cortex. J. Neurophysiol. 94: 734-740.

Orban, P., Rauchs, G., Balteau, E., Degueldre, C., Luxen, A., Maquet, P., and Peigneux, P. 2006. Sleep after spatial learning promotes covert reorganization of brain activity. Proc. Natl. Acad. Sci. 103: 7124-7129.

Packard, M.G. 1999. Glutamate infused posttraining into the hippocampus or caudate-putamen differentially strengthens place and response learning. Proc. Natl. Acad. Sci. 96: 12881-12886.

Piefke, M., Weiss, P.H., Zilles, K., Markowitsch, H.J., and Fink, G.R. 2003. Differential remoteness and emotional tone modulate the neural correlates of autobiographical memory. Brain 126: 650-668.

Piekema, C., Kessels, R.P., Mars, R.B., Petersson, K.M., and Fernández, G. 2006. The right hippocampus participates in short-term memory maintenance of object-location associations. Neuroimage 33: $374-382$.

Rasch, B., Buchel, C., Gais, S., and Born, J. 2007. Odor cues during slow-wave sleep prompt declarative memory consolidation. Science 315: $1426-1429$.

Ridderinkhof, K.R., Ullsperger, M., Crone, E.A., and Nieuwenhuis, S. 2004. The role of the medial frontal cortex in cognitive control. Science 306: 443-447.

Rudy, J.W., Biedenkapp, J.C., and O'Reilly, R.C. 2005. Prefrontal cortex and the organization of recent and remote memories: An alternative view. Learn. Mem. 12: 445-446.

Selemon, L.D. and Goldman-Rakic, P.S. 1988. Common cortical and subcortical targets of the dorsolateral prefrontal and posterior parietal cortices in the rhesus monkey: Evidence for a distributed neural network subserving spatially guided behavior. J. Neurosci. 8: $4049-4068$.

Sereno, M.I., Pitzalis, S., and Martinez, A. 2001. Mapping of contralateral space in retinotopic coordinates by a parietal cortical area in humans. Science 294: 1350-1354.

Sommer, T., Rose, M., Glascher, J., Wolbers, T., and Buchel, C. 2005. Dissociable contributions within the medial temporal lobe to encoding of object-location associations. Learn. Mem. 12: 343-351.

Stickgold, R. 2005. Sleep-dependent memory consolidation. Nature 437: $1272-1278$.

Sugiura, M., Sassa, Y., Watanabe, J., Akitsuki, Y., Maeda, Y., Matsue, Y., Fukuda, H., and Kawashima, R. 2006. Cortical mechanisms of person representation: Recognition of famous and personally familiar names. Neuroimage 31: 853-860.

Takashima, A., Petersson, K.M., Rutters, F., Tendolkar, I., Jensen, O., Zwarts, M.J., McNaughton, B.L., and Fernández, G. 2006. Declarative memory consolidation in humans: A prospective functional magnetic resonance imaging study. Proc. Natl. Acad. Sci. 103: $756-761$.

Takehara, K., Kawahara, S., and Kirino, Y. 2003. Time-dependent reorganization of the brain components underlying memory retention in trace eyeblink conditioning. J. Neurosci. 23: 9897-9905.

Teixeira, C.M., Pomedli, S.R., Maei, H.R., Kee, N., and Frankland, P.W. 2006. Involvement of the anterior cingulate cortex in the expression of remote spatial memory. J. Neurosci. 26: 7555-7564.

Tsao, D.Y., Freiwald, W.A., Tootell, R.B., and Livingstone, M.S. 2006. A cortical region consisting entirely of face-selective cells. Science 311: $670-674$

Voermans, N.C., Petersson, K.M., Daudey, L., Weber, B., Van Spaendonck, K.P., Kremer, H.P., and Fernández, G. 2004. Interaction between the human hippocampus and the caudate nucleus during route recognition. Neuron 43: 427-435.

Wagner, A.D., Maril, A., and Schacter, D.L. 2000. Interactions between forms of memory: When priming hinders new episodic learning. J. Cogn. Neurosci. 12 (Suppl 2): 52-60.

Walker, M.P., Brakefield, T., Hobson, J.A., and Stickgold, R. 2003. Dissociable stages of human memory consolidation and reconsolidation. Nature 425: 616-620.

Wallentin, M., Roepstorff, A., Glover, R., and Burgess, N. 2006. Parallel memory systems for talking about location and age in precuneus, caudate and Broca's region. Neuroimage 32: 1850-1864.

Received April 17, 2007; accepted in revised form May 7, 2007. 


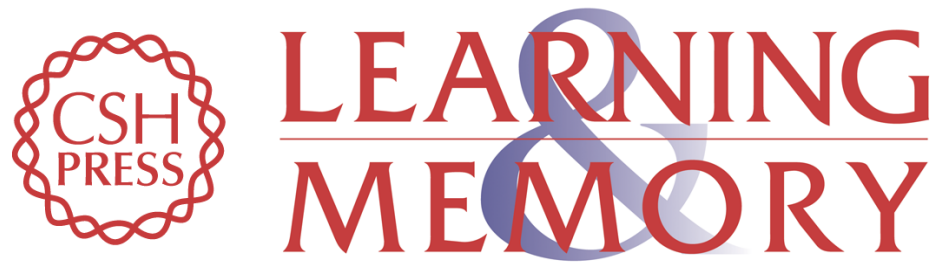

\section{Memory trace stabilization leads to large-scale changes in the retrieval network: A functional MRI study on associative memory}

Atsuko Takashima, Ingrid L.C. Nieuwenhuis, Mark Rijpkema, et al.

Learn. Mem. 2007, 14:

Access the most recent version at doi:10.1101//m.605607

References This article cites 47 articles, 19 of which can be accessed free at:

http://learnmem.cshlp.org/content/14/7/472.full.html\#ref-list-1

License

Email Alerting Receive free email alerts when new articles cite this article - sign up in the box at the Service top right corner of the article or click here. 